\title{
X-boson cumulant approach to the periodic Anderson model
}

\author{
R. Franco, M. S. Figueira \\ Instituto de Física, C.P. 100.093 \\ Universidade Federal Fluminense, UFF \\ Av. Litorânea s/no- 24210-340 Niterói, Rio de Janeiro, Brasil \\ M. E. Foglio \\ Instituto de Física "Gleb Wataghin" \\ Universidade Estadual de Campinas, UNICAMP \\ 13083-970 Campinas, São Paulo, Brasil
}

(October 25, 2018)

The Periodic Anderson Model (PAM) can be studied in the limit $U=\infty$ by employing the Hubbard $X$ operators to project out the unwanted states. We have already studied this problem employing the cumulant expansion with the hybridization as perturbation, but the probability conservation of the local states (completeness) is not usually satisfied when partial expansions like the "Chain Approximation (CHA)" are employed. Here we treat the problem by a technique inspired in the mean field approximation of Coleman's slave-bosons method, and we obtain a description that avoids the unwanted phase transition that appears in the mean-field slave-boson method both when $\mu>>E_{f}$ at low $T$ and for all parameters at intermediate temperatures.

71.10.-w, 71.27.+a, 71.28.+d, 75.20.Hr, 75.30.Mb

Short Title X-boson cumulant approach to the periodic Anderson model 


\section{INTRODUCTION}

In the limit of infinite Coulomb repulsion $(U=\infty)$ one can transform the Periodic Anderson Model (PAM) with two channel $(S U(2))$ by employing the Hubbard $X_{j, a b}=|j, a\rangle\langle j, b|$ operators, 1 where the set $\{|j, a\rangle\}$ is an orthonormal basis in the space of interest. Projecting out the components with more than one electron from any local state at site $j$ one obtains

$$
\begin{aligned}
H= & \sum_{\mathbf{k}, \sigma} E_{\mathbf{k}, \sigma} c_{\mathbf{k}, \sigma}^{\dagger} c_{\mathbf{k}, \sigma}+\sum_{j, \sigma} E_{f, j \sigma} X_{j, \sigma \sigma} \\
& +\sum_{j, \sigma, \mathbf{k}}\left(V_{j, \mathbf{k}, \sigma} X_{j, 0 \sigma}^{\dagger} c_{\mathbf{k}, \sigma}+V_{j, \mathbf{k}, \sigma}^{*} c_{\mathbf{k}, \sigma}^{\dagger} X_{j, 0 \sigma}\right) .
\end{aligned}
$$

The first term is the Hamiltonian of the conduction electrons (c-electrons). The second term describes independent localized electrons ( $f$-electrons), and a simple index $j$ is used to indicate the sites. The last term is the hybridization Hamiltonian giving the interaction between the $c$-electrons and the $f$-electrons with $V_{j, \mathbf{k}, \sigma}=\left(1 / \sqrt{N_{s}}\right) V_{\sigma}(\mathbf{k}) \exp \left(i \mathbf{k} \cdot \mathbf{R}_{j}\right)$, where $\mathbf{R}_{j}$ is the position of site $j$ and $N_{s}$ the number of sites. Notice that this interaction conserves the spin component $\sigma$.

The $X$ operators do not satisfy the usual commutation relations. One has then to use the product rules:

$$
X_{j, a b} \cdot X_{j, c d}=\delta_{b, c} X_{j, a d}
$$

and the diagrammatic methods based on Wick's theorem are therefore not applicable. We shall use instead a cumulant expansion that was originally employed by Hubbardl to study his model, and that was latter extended to the PAM.

There are four local states at each site $j$ of the lattice: the vacuum state $|j, 0\rangle$, the two states $|j, \sigma\rangle$ of one electron with spin component $\sigma$, and the state $|j, 2\rangle$ with two local electrons. When $U \rightarrow \infty$ the state $|j, 2\rangle$ is empty, and we have used the Hubbard operators to project it out from the space of local states at site $j$. In this space, the identity $I_{j}$ at site $j$ should then satisfy the relation:

$$
X_{j, 00}+X_{j, \sigma \sigma}+X_{j, \overline{\sigma \sigma}}=I_{j}
$$

where $\bar{\sigma}$ is the spin component opposite to $\sigma$, and the three $X_{j, a a}$ are the projectors into $|j, a\rangle$. The occupation numbers $n_{j, a}=\left\langle X_{j, a a}\right\rangle$ can be calculated from appropriate Green's functions (GF), and assuming translational invariance we can write $n_{j, a}=n_{a}$ (independent of $\mathrm{j}$ ), so that from Eq. (3) it follows that

$$
n_{0}+n_{\sigma}+n_{\bar{\sigma}}=1 \text {. }
$$

We shall call this relation "completeness", and it has been found that it is not usually satisfied when the $n_{a}$ are calculated with approximate cumulant Green's functions (GF) An approximation displaying this behavior is the "Chain Approximation" (CHA), which was first employed by Hewson, 5,6 and is the most general cumulant expansion with onfsecond order cumulants, as well as being $\Phi$-derivable. 4 In a previous work 9 we have stated a conjecture that gives a systematic way of achieving completeness by adding a set of diagrams to an arbitrary family. This result was verified for the CHA, and the resulting set of diagrams is not $\Phi$-derivable any more.

In the present work we recover completeness by a procedure similar to that employed by Coleman 9 in the slave boson approach. This last method predicts unphysical second order phase transitions both for all parameters at intermediate temperatures $T$ and in the Kondo region $\left(\mu>>E_{f, j \sigma}=E_{f}\right)$ at low $T$. Coleman 10 has observed that these effects are artifacts of the theory, and the advantage of the present treatment is that those spurious phase transitions do not occur. Our method gives results very close to those obtained by the slave boson in the Kondo limit at low temperatures, while it recovers those of the CHA at high $T$ for all parameters.

\section{X-BOSON CUMULANT METHOD}

The present work is a modification of a preliminary version 11 that was inspired in the mean-field approximation of Coleman's "slave boson" method. 12 He writes the Hubbard X operators as a product of ordinary bosons and fermions: $X_{j, o o} \rightarrow b_{j}^{+} b_{j}, X_{j, o \sigma} \rightarrow b_{j}^{+} f_{j, \sigma}, X_{j, \sigma o} \rightarrow$ $f_{j, \sigma}^{+} b_{j}$, and uses the equivalent of our Eq. (4) to avoid states with more than one electron at each site $j$. In the spirit of the mean field approximation $b_{i}^{+} \rightarrow<b_{i}^{+}>=r$, and the method of Lagrangian multipliers is then used to find the "best" Hamiltonian that satisfies Eq. (4). The problem is then reduced to an uncorrelated Anderson lattice with renormalized hybridization $V \rightarrow r V$ and $f$ level $\epsilon_{f} \rightarrow \epsilon_{f}+\lambda$.

Our method consists in adding the product of each Eq. (3) times a Lagrange multiplier $\Lambda_{j}$ to Eq. (1), and the new Hamiltonian generates the functional that we shall minimize employing Lagrange's method. Instead of the parameter $r$ we introduce

$$
R \equiv<X_{j, o o}>=<b_{j}^{+} b_{j}>,
$$

and we call the method "X-boson" because the Hubbard operator $X_{j, o o}$ has a "Bose-like" character." Considering Eqs. (3,5), and employing a constant hybridization $V$, as well as site independent local energies $E_{f, j, \sigma}=$ $E_{f, \sigma}$ and Lagrange parameters $\Lambda_{j}=\Lambda$, we obtain a new Hamiltonian with the same form of Eq. (11) 


$$
\begin{aligned}
H= & \sum_{\mathbf{k}, \sigma} E_{\mathbf{k}, \sigma} c_{\mathbf{k}, \sigma}^{\dagger} c_{\mathbf{k}, \sigma} \\
& +\sum_{j, \sigma} \tilde{E}_{f, \sigma} X_{j, \sigma \sigma}+N_{s} \Lambda(R-1) \\
& +V \sum_{j, \mathbf{k}, \sigma}\left(X_{j, 0 \sigma}^{\dagger} c_{\mathbf{k}, \sigma}+c_{\mathbf{k}, \sigma}^{\dagger} X_{j, 0 \sigma}\right),
\end{aligned}
$$

but with a renormalized localized energy

$$
\tilde{E}_{f, \sigma}=E_{f, \sigma}+\Lambda \text {. }
$$

This procedure allows for an independent variation of $R$ when the Free Energy is minimized, even though the completeness relation

$$
R=1-\sum_{\sigma}<X_{\sigma \sigma}>
$$

must be simultaneously satisfied.

In the slave-boson approach a one-body Hamiltonian is obtained at this stage, and Eq. (4) is then necessary to avoid the occupation of more than one localized electron per site, while the conservation of probability in the space of the local states is automatically satisfied, because normal fermion operators are employed in the transformed Hamiltonian. The Eq. (6) on the other hand employs $X$-operators, that force the local states to be singly occupied at most, but Eq. (位) (completeness) must be imposed here because it is not automatically satisfied when approximate GF are employed to calculate the $n_{a}$. In the present work we shallemploy the GF of the Chain approximation (CHA), because they give a fair description of the system in spite of their simplicity.

The present treatment employs the Grand Canonical Ensemble of electrons, and instead of Eq. (11) we shall use

$$
\mathcal{H}=H-\mu\left\{\sum_{\mathbf{k}, \sigma} c_{\mathbf{k}, \sigma}^{\dagger} c_{\mathbf{k}, \sigma}+\sum_{j a} \nu_{a} X_{j, a a}\right\} \quad,
$$

where $\nu_{a}=0,1$ is the number of electrons in state $\mid a>$. It is then convenient to define

$$
\varepsilon_{j, a}=E_{f, j, a}-\mu \nu_{a}
$$

and

$$
\varepsilon_{\mathbf{k} \sigma}=E_{\mathbf{k} \sigma}-\mu,
$$

because $E_{f, j, a}$ and $E_{\mathbf{k}, \sigma}$ appear only in that form in all the calculations. The exact and unperturbed averages of the operator $A$ are denoted in what follows by $<A>_{\mathcal{H}}$ and $<A>$ respectively.

\section{THE CHAIN APPROXIMATION GREEN'S FUNCTIONS}

The CHA gives simple but useful approximate propagators, obtained in the cumulant expansion by taking the infinite sum of all the diagrams that contain ionic vertices with only two lines. The laborious calculation of the general treatment is rather simplified in this case, and we shall give a brief description of the technique, particularly when only the imaginary time is Fourier transformed, because this intermediate situation is not discussed in reference 11 and it is necessary to calculate the impurity problem. The only $X_{\alpha}$ and $X_{\alpha}^{\dagger}$ operators of the Fermi type that appear in the calculation have $\alpha=(0, \sigma)$, and the f-electron GF in real space and imaginary time are

$$
G^{f f}(j, \alpha, \tau ; j \prime, \alpha \prime, \tau \prime)=\left\langle\left(X_{j, \alpha}(\tau) X_{j \prime, \alpha \prime}^{\dagger}(\tau \prime)\right)_{+}\right\rangle_{\mathcal{H}(12)}
$$

where $\widehat{X}_{j, \alpha}(\tau)=\exp (\tau \mathcal{H}) X_{j, \alpha} \exp (-\tau \mathcal{H})$ corresponds to the Heisenberg representation and the subindex + indicates that the operators inside the parenthesis are taken in the order of increasing $\tau$ to the left, with a change of sign when the two Fermi-type pperators have to be exchanged to obtain this ordering. In a similar way one defines the c-electron GF $G^{c c}(\mathbf{k}, \sigma, \tau ; \mathbf{k} /, \sigma \prime, \tau \prime)$ as well as the mixed GF $G^{f c}(j, \alpha, \tau ; \mathbf{k} \prime, \sigma \prime, \tau \prime)$ and $G^{c f}(\mathbf{k}, \sigma, \tau ; j \prime, \alpha \prime, \tau \prime)$.

The boundary conditions of these GF with respect to $\tau$ makes possible to expand them in Fourier seriest employing the Matsubara frequencies $\omega_{n}=(2 n+1) i \pi / \beta$, where $n$ is any integer. Because of the invariance of $\mathcal{H}$ against $\tau$ translations we have frequency conservation, so that $G^{f f}\left(j, \alpha, i \omega_{n} ; j \prime, \alpha \prime, i \omega_{n}^{\prime}\right)=0$ unless $\omega_{n}+\omega_{n}^{\prime}=0$, and one can then write

$$
G^{f f}\left(j, \alpha, i \omega_{n} ; j^{\prime}, \alpha \prime, i \omega_{n}^{\prime}\right)=G_{j \alpha ; j \prime \alpha \prime}^{f f}\left(z_{n}\right) \Delta\left(\omega_{n}+\omega_{n^{\prime}}\right)
$$

and similar relations for the $G^{c c}, G^{f c}$, and $G^{c f}$. Here we have used $z_{n} \equiv i \omega_{n}$, and we shall keep this notation in what follows, as well as employing $\Delta(\gamma) \equiv \delta_{\gamma, 0}$ (a modified notation for Krönecker's delta). The "bare GF" (i.e. with all $V_{j, \mathbf{k}, \sigma}=0$ ) take a fairly simple form because many of the relevant operators become statistically independent, and we then have

$$
\begin{aligned}
& G^{f f}\left(j, \alpha, z_{n} ; j \prime, \alpha \prime, z_{n}^{\prime}\right) \Longrightarrow \\
& \quad G_{f, \alpha}^{0}\left(z_{n}\right) \Delta\left(\omega_{n}+\omega_{n}\right)^{\prime} \delta_{j, j \prime} \delta_{\alpha, \alpha \prime}, \\
& G^{c c}\left(\mathbf{k}, \alpha, z_{n} ; \mathbf{k} \prime, \alpha \prime, z_{n^{\prime}}\right) \Longrightarrow \\
& \quad G_{c, \mathbf{k} \sigma}^{0}\left(z_{n}\right) \Delta\left(\omega_{n}+\omega_{n^{\prime}}\right) \delta_{\mathbf{k}, \mathbf{k} \prime} \delta_{\sigma, \sigma \prime} \\
& G^{f c} \Longrightarrow G^{c f} \Longrightarrow 0
\end{aligned}
$$

The $\delta_{\alpha, \alpha}$ in the bare GF follows from the commutation of $\mathcal{H}$ with the $z$ component of the spin.

The diagrams in real space that contribute to the CHA are schematized in Fig (1). The meaning of the symbols in the cumulant diagrams is the following:

a) the "vertex" $=G_{f, 0 \sigma}^{o}\left(z_{n}\right)=-D_{0 \sigma} /\left(z_{n}-\varepsilon_{f, \sigma}\right)$ is the $f$ bare cumulant $\mathrm{GF}$, where $D_{0 \sigma}=<X_{o o}>+<X_{\sigma \sigma}>$

b) the "vertex" $\bigcirc=G_{c, \mathbf{k} \sigma}^{o}\left(z_{n}\right)=-1 /\left(z_{n}-\varepsilon_{\mathbf{k} \sigma}\right)$ 
is the $c$ bare cumulant GF

c) the lines (edges) determine an open loop with a definite direction. When the line points to the f-vertex is $\longleftarrow=V_{j, \mathbf{k}, \sigma}$, while $\longleftarrow=V_{j, \mathbf{k}, \sigma}^{*}$ when it points to the conduction vertex

d) The cumbersome sign and symmetry factors 1 . 3 are rather simple in the CHA: it is only necessary to multiply the $G^{f c}$ and the $G^{c f}$ into a minus sign.

e) As both the $\omega$ and the $\sigma$ are conserved along the open loop, it only remains to sum over all the internal $j$ and k.
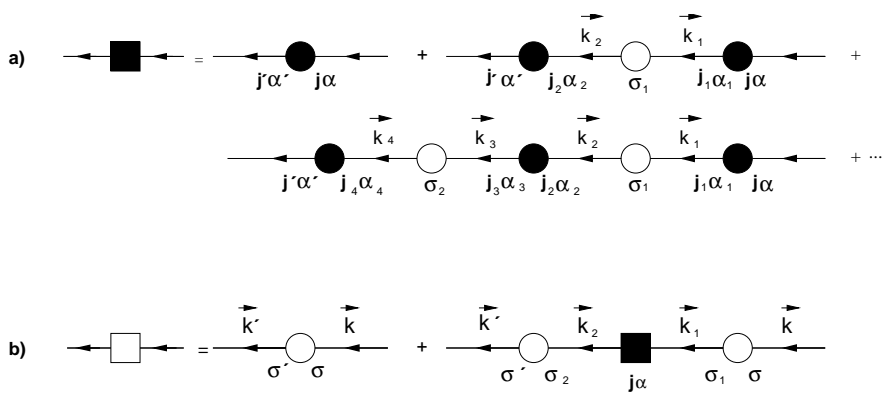

c)

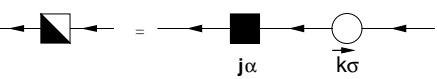

FIG. 1. The Green's function diagrams in the CHA. The filled circles (vertices) corresponds to the $f$-electron cumulants and the empty ones to those of the $c$-electrons. The lines (edges) joining two vertices represent the perturbation (hybridization) (cf. Section III) a) Diagrams for the $f$-electron GF in the CHA. The CHA diagram for the $f$-electron $G_{j_{i} \alpha_{i}, j_{f} \alpha_{f}}^{f f}\left(i \omega_{n}\right)$ is represented by the filled square to the left. b) Diagrams for the $c$ electron GF in the CHA. The $G_{\mathbf{k} \prime, \sigma / ; \mathbf{k}, \sigma}^{c c}\left(z_{n}\right)$ is represented by the square symbol to the left. c) Diagrams for the $f$-c electron GF in the CHA. The $G_{\mathbf{j} ; \mathbf{k}, \sigma}^{f c}\left(z_{n}\right)$ is represented by the square symbol to the left.

We then obtain (with $\alpha=(0 \sigma)$ ):

$$
\begin{aligned}
& G_{j \prime \alpha \prime ; j \alpha}^{f f}\left(z_{n}\right)=\delta_{\alpha \prime, \alpha} G_{f, \alpha}^{o}\left(z_{n}\right) \\
& +G_{f, \alpha}^{o}\left(z_{n}\right) \sum_{\mathbf{k}_{1}} V_{j \prime, \mathbf{k}_{1} \sigma} G_{c, \mathbf{k}_{1} \sigma}^{o}\left(z_{n}\right) V_{j, \mathbf{k}_{1} \sigma}^{*} G_{f, \alpha}^{o}\left(z_{n}\right) \\
& +G_{f, \alpha}^{o}\left(z_{n}\right) \sum_{\mathbf{k}_{2}} V_{j \prime, \mathbf{k}_{2} \sigma} G_{c, \mathbf{k}_{2} \sigma}^{o}\left(z_{n}\right) \sum_{j_{1}} V_{j_{1}, \mathbf{k}_{2} \sigma}^{*} G_{f, \alpha}^{o}\left(z_{n}\right) \\
& \times \sum_{\mathbf{k}_{1}} V_{j_{1}, \mathbf{k}_{1} \sigma} G_{c, \mathbf{k}_{1} \sigma}^{o}\left(z_{n}\right) V_{j, \mathbf{k}_{1} \sigma}^{*} G_{f, \alpha}^{o}\left(z_{n}\right)+\ldots
\end{aligned}
$$

\section{The impurity case}

When there is a single impurity at a given $j$, each of

the sums over the internal $j_{r}$ reduces to a single term with $j \prime=j$, and we introduce

$$
\begin{aligned}
M_{\sigma}\left(z_{n}\right) & =\sum_{\mathbf{k}_{1}} V_{j, \mathbf{k}_{1} \sigma} G_{c, \mathbf{k}_{1} \sigma}^{o}\left(z_{n}\right) V_{j, \mathbf{k}_{1} \sigma}^{*} \\
& =\frac{1}{N_{s}} \sum_{\mathbf{k}}\left|V_{\sigma}(\mathbf{k})\right|^{2} G_{c, \mathbf{k} \sigma}^{o}\left(z_{n}\right),
\end{aligned}
$$

which is the local GF at site $j$ times $\left|V_{\sigma}\right|^{2}$ when the hybridization is purely local, i.e. when $V_{\sigma}(\mathbf{k})=V_{\sigma}$. The Eq. (17) is then a geometric series that is easily summed:

$$
\begin{gathered}
G_{j \prime \alpha \prime ; j \alpha}^{f f}\left(z_{n}\right)=\delta_{\alpha \prime, \alpha} \delta_{j \prime, j} \frac{G_{f, \alpha}^{o}\left(z_{n}\right)}{1-G_{f, \alpha}^{o}\left(z_{n}\right) M_{\sigma}\left(z_{n}\right)} \\
=\delta_{\alpha \prime, \alpha} \delta_{j \prime, j} \frac{-D_{0 \sigma}}{z_{n}-\varepsilon_{f, \sigma}+D_{0 \sigma} M_{\sigma}\left(z_{n}\right)} .
\end{gathered}
$$

Employing the same technique, we obtain for the CHA the $G^{c c}$, that gives the scattering by the local impurity of a conduction electron $\mathbf{k}, \sigma$ into $\mathbf{k} /, \sigma /$ :

$$
\begin{aligned}
& G_{\mathbf{k} / \sigma \prime ; \mathbf{k} \sigma}^{c c}\left(z_{n}\right)=\delta_{\sigma \prime, \sigma}\left\{G_{c, \mathbf{k} \sigma}^{o}\left(z_{n}\right) \delta_{\mathbf{k}, \mathbf{k} \prime}\right. \\
& \left.+G_{c, \mathbf{k} / \sigma}^{o}\left(z_{n}\right) V_{\sigma}^{*}(\mathbf{k} \prime) G_{j \alpha ; j \alpha}^{f f}\left(z_{n}\right) \times V_{\sigma}(\mathbf{k}) G_{c, \mathbf{k} \sigma}^{o}\left(z_{n}\right)\right\},
\end{aligned}
$$

as well as the mixed GF (with $\left.\alpha^{\prime}=\left(0 \sigma^{\prime}\right)\right)$

$$
G_{j \prime \alpha \prime ; \mathbf{k} \sigma}^{f c}\left(z_{n}\right)=\delta_{\sigma /, \sigma} \delta_{j \prime, j} G_{j ; \mathbf{k} \sigma}^{f c}\left(z_{n}\right),
$$

where we have already included the minus sign (discussed in rule d) above) into

$$
\begin{aligned}
& G_{j ; \mathbf{k} \sigma}^{f c}\left(z_{n}\right)=-G_{j \alpha ; j \alpha}^{f f}\left(z_{n}\right) V_{j, \mathbf{k} \sigma} G_{c, \mathbf{k} \sigma}^{o}\left(z_{n}\right) \\
= & -\frac{D_{0 \sigma} V_{j, \mathbf{k} \sigma}}{z_{n}-\varepsilon_{f, \sigma}+D_{0 \sigma} M_{\sigma}\left(z_{n}\right)} \times \frac{1}{z_{n}-\varepsilon_{\mathbf{k} \sigma}} .
\end{aligned}
$$

\section{The lattice case}

The case of the GF in reciprocal space and imaginary frequencies has been discussed in detail in reference, 1 and in the CHA one follows the same prescriptions given above, but replacing the sum over internal $j$ and $\mathbf{k}$ by a conservation of $\mathbf{k}$ along the whole chain, so that we have

$$
\begin{aligned}
& G^{f f}\left(\mathbf{k} /,(0 \sigma \prime), z_{n} \prime ; \mathbf{k},(0 \sigma), z_{n}\right) \\
& =\delta_{\mathbf{k}, \mathbf{k} \prime} \delta_{\sigma, \sigma \prime} \Delta\left(\omega_{n}+\omega_{n}\right) G_{\mathbf{k} \sigma}^{f f}\left(z_{n}\right), \\
& G^{c c}\left(\mathbf{k} \prime, \sigma \prime, z_{n}^{\prime \prime} ; \mathbf{k}, \sigma, z_{n}\right) \\
& =\delta_{\mathbf{k}, \mathbf{k} \prime} \delta_{\sigma, \sigma \prime} \Delta\left(\omega_{n}+\omega_{n}^{\prime}\right) G_{\mathbf{k} \sigma}^{c c}\left(z_{n}\right), \\
& G^{f c}\left(\mathbf{k} \prime,(0 \sigma \prime), z_{n} \prime ; \mathbf{k}, \sigma, z_{n}\right) \\
& =\delta_{\mathbf{k}, \mathbf{k} \prime} \delta_{\sigma, \sigma \prime} \Delta\left(\omega_{n}+\omega_{n}{ }^{\prime}\right) G_{\mathbf{k} \sigma}^{f c}\left(z_{n}\right),
\end{aligned}
$$

where

$$
G_{\mathbf{k} \sigma}^{f f}\left(z_{n}\right)=\frac{-D_{\sigma}\left(z_{n}-\varepsilon_{\mathbf{k} \sigma}\right)}{\left(z_{n}-\varepsilon_{f, \sigma}\right)\left(z_{n}-\varepsilon_{\mathbf{k} \sigma}\right)-\left|V_{\sigma}(\mathbf{k})\right|^{2} D_{\sigma}},
$$




$$
G_{\mathbf{k} \sigma}^{c c}\left(z_{n}\right)=\frac{-\left(z_{n}-\varepsilon_{f, \sigma}\right)}{\left(z_{n}-\varepsilon_{f, \sigma}\right)\left(z_{n}-\varepsilon_{\mathbf{k} \sigma}\right)-\left|V_{\sigma}(\mathbf{k})\right|^{2} D_{\sigma}},
$$

and

$$
G_{\mathbf{k} \sigma}^{f c}\left(z_{n}\right)=\frac{-D_{\sigma} V_{\sigma}(\mathbf{k})}{\left(z_{n}-\varepsilon_{f, \sigma}\right)\left(z_{n}-\varepsilon_{\mathbf{k} \sigma}\right)-\left|V_{\sigma}(\mathbf{k})\right|^{2} D_{\sigma}} .
$$

\section{The Slave Boson GF vs the GF of the CHA}

The Slave Boson GF in the mean field approximation (cf. references 12 14) are just the GF of the uncorrelated problem $(U=0)$ but with a renormalized hybridization $V \rightarrow \bar{V} \equiv r V$ plus a condition that forces the local electron to an occupation less or equal to one. The GF of the CHA given above are formally very close to the uncorrelated ones, but they can not be reduced to them by any change of scale (except for $D_{\sigma}=1$, when we recover the slave-boson Green's functions if we put $V \rightarrow r V=\bar{V}$ in Eqs. (19 24) and Eqs. (25)30)). The obvious change $\sqrt{D_{0 \sigma}} V \rightarrow V$, leaves an extra factor $D_{0 \sigma}$ in the $G_{j \prime \alpha \prime ; j \alpha}^{f f}\left(z_{n}\right)$ and $G_{\mathbf{k} \sigma}^{f f}\left(z_{n}\right)$, as well as a $\sqrt{D_{0 \sigma}}$ in both $G_{\mathbf{j} ; \mathbf{k}, \sigma}^{f c}\left(z_{n}\right)$ and $G_{\mathbf{k} \sigma}^{f c}\left(z_{n}\right)$, and these factors are responsible for the correlation in this approximation, and lead to essential differences with the uncorrelated case.13 In particular, they force the total occupation $n_{f}$ of the $\mathrm{f}$ electron to $n_{f} \leq 1$, while in the uncorrelated case the relation $n_{f} \leq 2$ is satisfied. In the slave boson method the imposed condition $n_{f} \leq 1$ is fulfilled by a shift in the local energy $\varepsilon_{f, \sigma} \rightarrow \widetilde{\varepsilon}_{f, \sigma} \equiv \varepsilon_{f, \sigma}+\lambda$ and a reduction of the hybridization to $V \rightarrow \widetilde{V} \equiv r V$. From an operational point of view, a shift in $\varepsilon_{f, \sigma}$ might not be sufficient to force $n_{f} \leq 1$ because the hybridization extends the $\mathrm{f}$ spectral density to the whole conduction band, and reducing $V$ helps to satisfy this condition. By increasing the temperature $T$ or the chemical potential $\mu$ the value $\widetilde{V}=0$ is presently reached, leading to a decoupling of the two type of electrons that can be interpreted, from a more general point of view, as a change of phase related to a symmetry breaking of the mean field Hamiltonian. Although the condition that forces completeness in the CHA is identical to that employed in the slave boson method to force $n_{f} \leq 1$, it has a rather different origin, being only a consequence of using areduced set of diagrams in the perturbative expansion, 8 and the departures from completeness are usually very moderate. In the formalism described in the present work, it is this essential difference between the two methods that eliminates the spurious phase transitions appearing in the slave boson method.

\section{THE SINGLE IMPURITY PROBLEM}

In the X-boson approach $D_{\sigma}=R+n_{f \sigma}$ must be calculated self-consistently through minimization with respect to the parameter $R$ of an adequate thermodynamic potential. When the total number of electrons $N_{t}$, the temperature $T$ and the volume $V_{s}$ are kept constant one should minimize the Helmholtz free energy, but the same minimum is obtained by employing the thermodynamic potential $\Omega=-k_{B} T \ln (\mathcal{Q})$, (where $\mathcal{Q}$ is the Grand Partition Function) and keeping $T, V_{s}$, and the chemical potential $\mu$ constant (this result is easily obtained by employing standard thermodynamic techniques).

A convenient way of calculating $\Omega$ is to employ the $\xi$ parameter integration method 15, 26,27 This method introduces a $\xi$ dependent Hamiltonian $H(\xi)=H_{o}+\xi H_{1}$ through a coupling constant $\xi$ (with $0 \leq \xi \leq 1$ ), where $H_{1}$ is the hybridization in our case. For each $\xi$ there is an associated thermodynamic potential $\Omega(\xi)$ which satisfies:

$$
\left(\frac{\partial \Omega}{\partial \xi}\right)_{V_{s}, T, \mu}=<H_{1}(\xi)>_{\xi},
$$

where $\langle A\rangle_{\xi}$ is the ensemble average of an operator $A$ for a system with Hamiltonian $H(\xi)$ and the given values of $\mu, T$, and $V_{s}$. Integrating this equation gives

$$
\Omega=\Omega_{o}+\int_{0}^{1} d \xi<H_{1}(\xi)>_{\xi},
$$

where $\Omega_{o}$ is the thermodynamic potential of the system with $\xi=0$. This value of $\xi$ corresponds to a system without hybridization, and one obtains (in the absence of magnetic field $\varepsilon_{\mathbf{k} \sigma}=\varepsilon_{\mathbf{k}}$ and $\widetilde{\varepsilon}_{f \sigma}=\widetilde{\varepsilon}_{f}$ )

$$
\begin{aligned}
\Omega_{o}= & -\frac{2}{\beta} \sum_{\mathbf{k}} \ln \left[1+\exp \left(-\beta \varepsilon_{\mathbf{k}}\right)\right] \\
& -\frac{1}{\beta} \ln \left[1+2 \exp \left(-\beta \widetilde{\varepsilon}_{f}\right)\right]+\Lambda(R-1),
\end{aligned}
$$

and to calculate $\Omega$ in Eq.(32) we use

$$
<H_{1}>_{\xi}=2 R e\left[\sum_{\mathbf{k} \sigma} V_{j, \mathbf{k}, \sigma}^{*}\left\langle c_{\mathbf{k} \sigma}^{\dagger} X_{0 \sigma}\right\rangle_{\xi}\right] .
$$

The average $\left\langle c_{\mathbf{k} \sigma}^{\dagger} X_{0 \sigma}\right\rangle_{\xi}$ is obtained from the analytical continuation of the Matsubara $G_{\mathbf{j} ; \mathbf{k}, \sigma}^{f c}\left(z_{n}, \xi\right) \rightarrow$ $\bar{G}_{\mathbf{j} ; \mathbf{k}, \sigma}^{f c}(z, \xi)$ into the complex upper and lower semiplanes, where $G_{\mathbf{j}: \mathbf{k}, \sigma}^{f c}\left(z_{n}, \xi\right)$ is the GF in Eq. 23) but for $V_{j, \mathbf{k}, \sigma} \rightarrow \xi V_{j, \mathbf{k}, \sigma}$. One then finds

$$
\begin{aligned}
& \left\langle c_{\mathbf{k}, \sigma}^{\dagger} X_{j, 0 \sigma}\right\rangle_{\xi}=\frac{i}{2 \pi} \int_{-\infty}^{\infty} d \omega n_{F}(\omega) \\
& \times\left\{\bar{G}_{j, \mathbf{k} \sigma}^{f c}(\omega+i 0 ; \xi)-\bar{G}_{j, \mathbf{k} \sigma}^{f c}(\omega-i 0 ; \xi)\right\}
\end{aligned}
$$


where $n_{F}(x)=1 /[1+\exp (\beta x)]$ is the Fermi-Dirac distribution. From Eqs. (18,34,35) we then obtain

$$
\begin{aligned}
\left\langle H_{1}\right\rangle_{\xi}= & -\frac{1}{\pi} \int_{-\infty}^{\infty} d \omega n_{F}(\omega) \\
& \times \sum_{\sigma} \operatorname{Im}\left\{\frac{\xi D_{0 \sigma} M_{\sigma}\left(\omega^{+}\right)}{\omega^{+}-\widetilde{\varepsilon}_{f}+D_{0 \sigma} \xi^{2} M_{\sigma}\left(\omega^{+}\right)}\right\},
\end{aligned}
$$

where $\omega^{+}=\omega+i 0$. This equation has an interesting scaling property: it is equal to the corresponding expression of the uncorrelated system for the scaled parameter $\bar{V}_{j, \mathbf{k}, \sigma}=\sqrt{D_{0 \sigma}} V_{j, \mathbf{k}, \sigma}$ (it is enough to remember that by replacing $D_{0 \sigma}=1$ in the $\mathrm{GF}$ of the $\mathrm{CHA}$ one obtains the corresponding GF of the uncorrelated system). Rather than performing the $\xi$ and $\omega$ integrations, we shall use the value of the $\Omega^{u}$ for the uncorrelated system with $\bar{V}_{j, \mathbf{k}, \sigma}=\sqrt{D_{0 \sigma}} V_{j, \mathbf{k}, \sigma}$ and employ Eq. (32) to calculate $\int_{0}^{1} d \xi<H_{1}^{u}(\xi)>_{\xi}=\Omega^{u}-\Omega_{o}^{u}$, where

$\Omega_{o}^{u}=\frac{-2}{\beta}\left[\sum_{\mathbf{k}} \ln \left[1+\exp \left(-\beta \varepsilon_{\mathbf{k}}\right)\right]+\ln \left[1+\exp \left(-\beta \widetilde{\varepsilon}_{f}\right)\right]\right]$ $+\Lambda(R-1)$

is the $\Omega^{u}$ for $\bar{V}_{j, \mathbf{k}, \sigma}=0$. In our case the unperturbed Hamiltonian for the lattice problem is

$$
\begin{aligned}
& H^{u}=\sum_{\mathbf{k}, \sigma} \varepsilon_{\mathbf{k}, \sigma} c_{\mathbf{k}, \sigma}^{\dagger} c_{\mathbf{k}, \sigma} \\
& +\sum_{j, \sigma} \widetilde{\varepsilon}_{f} f_{j, \sigma}^{\dagger} f_{j, \sigma}+N_{s} \Lambda(R-1) \\
& +\sum_{j, \mathbf{k}, \sigma}\left(\bar{V}_{j, \mathbf{k}, \sigma} f_{j, \sigma}^{\dagger} c_{\mathbf{k}, \sigma}+\bar{V}_{j, \mathbf{k}, \sigma}^{*} c_{\mathbf{k}, \sigma}^{\dagger} f_{j, \sigma}\right),
\end{aligned}
$$

and in the impurity case the sum over sites reduces to the impurity site and $N_{s} \Lambda(R-1) \rightarrow \Lambda(R-1)$. This Hamiltonian can be easily diagonalized, and the corresponding $\mathcal{H}^{u}$ can be written

$$
\mathcal{H}^{u}=\sum_{i, \sigma} \omega_{i, \sigma} \alpha_{i, \sigma}^{\dagger} \alpha_{i, \sigma}+\Lambda(R-1)
$$

where $\alpha_{i, \sigma}^{\dagger}\left(\alpha_{i, \sigma}\right)$ are the creation (destruction) operators of the composite particles of energies $\omega_{i, \sigma}$ (there are $N_{s}+$ 1 states for each spin $\sigma$ ). The calculation of

$$
\Omega^{u}=\frac{-1}{\beta} \sum_{i, \sigma} \ln \left[1+\exp \left(-\beta \omega_{i, \sigma}\right)\right]+\Lambda(R-1),
$$

is straightforward, and employing Eqs. (32, 33, 37, 40 we find

$$
\Omega=\bar{\Omega}_{0}+\frac{-1}{\beta} \sum_{i, \sigma} \ln \left[1+\exp \left(-\beta \omega_{i, \sigma}\right)\right]+\Lambda(R-1),
$$

where

$$
\bar{\Omega}_{0} \equiv \Omega_{o}-\Omega_{o}^{u}=-\frac{1}{\beta} \ln \left[\frac{1+2 \exp \left(-\beta \widetilde{\varepsilon}_{f}\right)}{\left(1+\exp \left(-\beta \widetilde{\varepsilon}_{f}\right)\right)^{2}}\right],
$$

and the eigenvalues $\omega_{i, \sigma}$ of the $\mathcal{H}^{u}$ are just given by the poles of the GF in the CHA (Eq. (19)).

It is interesting that all the correlation effects on the thermodynamic potential appear in the $\Omega_{o}$, that corresponds to the unperturbed system. The effect of the perturbation in the present approximation is to redistribute the energy of the quasi-particles in the same way as in an uncorrelated system with hybridization constant $\bar{V}_{j, \mathbf{k}, \sigma}=\sqrt{D_{0 \sigma}} V_{j, \mathbf{k}, \sigma}$, and one could then expect Fermi liquid behavior in the CHA (see a formal discussion in the Appendix).

The parameter $\Lambda$ is obtained minimizing $\Omega$ with respect to $R^{14}$ (at constant $\mu$ as discussed at the beginning of this Section). To simplify the calculations we shall consider a conduction band with a constant density of states and width $W=2 D$, and an hybridization constant $V_{\sigma}(\mathbf{k})=V$. We then obtain

$$
\frac{\partial \Omega}{\partial R}=\sum_{i, \sigma} n_{F}\left(\omega_{i, \sigma}\right)\left(\frac{\partial \omega_{i, \sigma}}{\partial R}\right)+\Lambda=0 .
$$

The poles of the impurity GF satisfy:

$$
\omega_{i, \sigma}=\varepsilon_{f}+\left(\frac{V^{2} D_{\sigma}}{2 D}\right) \ln \left[\frac{\omega_{i, \sigma}+D}{\omega_{i, \sigma}-D}\right],
$$

and calculating the $R$ derivative of $\omega_{i}(\vec{k}, \sigma)$ we obtain

$$
\Lambda=\frac{-V^{2}}{2 D} \sum_{i \sigma} \ln \left(\frac{\omega_{i, \sigma}+D}{\omega_{i, \sigma}-D}\right) \frac{\left(\omega_{i, \sigma}^{2}-D^{2}\right) n_{F}\left(\omega_{i, \sigma}\right)}{\left(\omega_{i, \sigma}^{2}-D^{2}+V^{2} D_{\sigma}\right)}
$$

In the absence of magnetic field, i.e. when the $\omega_{i, \sigma}=\omega_{i}$ is independent of $\sigma$, we can write this equation as:

$$
\begin{aligned}
\Lambda= & \frac{-V^{2}}{D} \int_{-\infty}^{\infty} d \omega \rho_{f}(\omega) \ln \left(\frac{\omega+D}{\omega-D}\right) \\
& \times \frac{\left(\omega^{2}-D^{2}\right) n_{F}(\omega)}{\left(\omega^{2}-D^{2}+V^{2} D_{\sigma}\right)},
\end{aligned}
$$

where $\rho_{f}(\omega)=\sum_{i} \delta\left(\omega-\omega_{i}\right)$ is the density of $f$ states, and the chemical potential $\mu$ of the electrons was included into the single particle energies (cf. Eqs. (10,11)). The Kondo temperature

$$
T_{K} \simeq D \exp \left(\frac{\widetilde{\varepsilon}_{f}}{\bar{V}^{2}}\right)
$$

is defined as the $T$ that makes the slave boson parameter $r$ to vanish, but another useful definition was given by Bernhard and Lacroix 18 by taking $T_{K}$ as the crossover temperature, determined by the maximum of the derivative of $\left\langle c_{i \sigma}^{\dagger} f_{i \sigma}\right\rangle$ with respect to $T$. 


\section{IMPURITY RESULTS}

The results presented in this section correspond to a Kondo regime with the following parameters: $E f=$ $-0.15 ; W=2.0, V=0.3$.

Figure 2 shows the evolution with $T$ of the parameter $R$, that measures the hole occupation, together with the corresponding $r^{2}$ of the usual mean field slave boson, as well as the two occupation number $n_{f}$. The figure shows that $r^{2} \rightarrow 0$ at a finite $T$ (Kondo temperature $T_{K}$ ) while $R$ remains positive, avoiding the spurious phase transition. The $n_{f} \rightarrow 1$ at $T_{K}$ for the slave boson, while it remains lower than 1 for the X-boson at all $T$.

At high temperatures the system is well described by localized moments coupled to the conduction electrons through a Coqblin-Schriefer type Hamiltonian, and even at high temperatures there should not be a complete decoupling nor a second order phase transition as suggested by the mean field slave-boson theory. 19 As the GF of the CHA retain the correlation properties of the system, the X-boson approach shows no decoupling nor spurious phase transitions, as discussed in Section III 3 . One can see in Figure 2 that the two approaches give similar results at low temperatures.

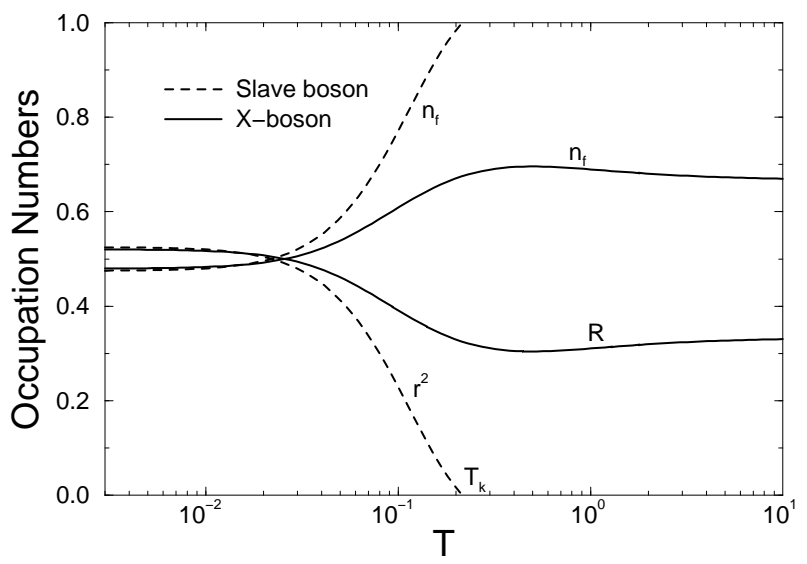

FIG. 2. Occupation numbers $n_{f}$, and parameters $r^{2}$ and $R$ as a function of $T$ for both the slave boson and the X-boson methods. The figures correspond to the following parameters: $E_{f}=-0.15 ; W=2.0 ; V=0.3 ; \mu=0.0$.

In Figure 3 we represent the parameters $\lambda$ and $\Lambda$ as a function of temperature. We observe that the slave boson $\lambda$ breaks down at the Kondo temperature $T_{K}$ whereas the $\mathrm{X}$-boson $\Lambda$ goes continuously to zero. In the high temperature limit the results obtained with the usual CHA are recovered by the X-boson. In Figure 4 we show the evolution of $\lambda, r^{2}$, and $n_{f}$, as a function of the chemical potential $\mu$ in the usual slave boson approach: the formalism breaks down at a value $\mu_{0}$, where $n_{f} \rightarrow 1$. In Figure 5 we show the evolution of the parameters $\Lambda, R$, and $n_{f}$ as a function of the chemical potential $\mu$ for the $\mathrm{X}$-boson approach. The results of the two approaches are similar for $\mu<\mu_{0}$, i.e. before the breakdown of the slave boson method (compare Fig. 1 and Fig. 5). The parameter $\Lambda$ reaches a maximum in the Kondo region and goes to zero when $\mu>>E_{f}$, where $n_{f} \rightarrow 1$, and in this limit we recover the usual CHA.

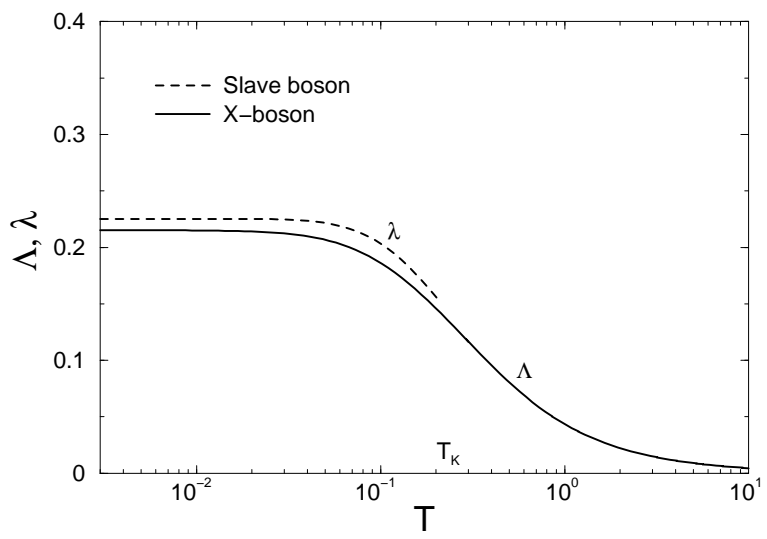

FIG. 3. Evolution with temperature $T$ of the renormalizing $\Lambda$ and $\lambda$, for the same model parameters used in Figure 2.

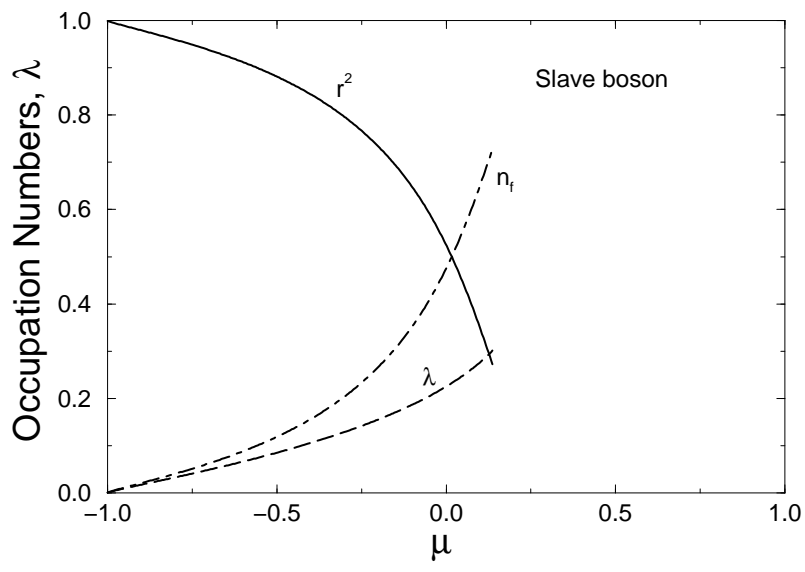

FIG. 4. Slave boson: $\lambda, r^{2}$, and $n_{f}$ vs. $\mu$ for the same model parameters used in Figure 2 and $T=0.001$.

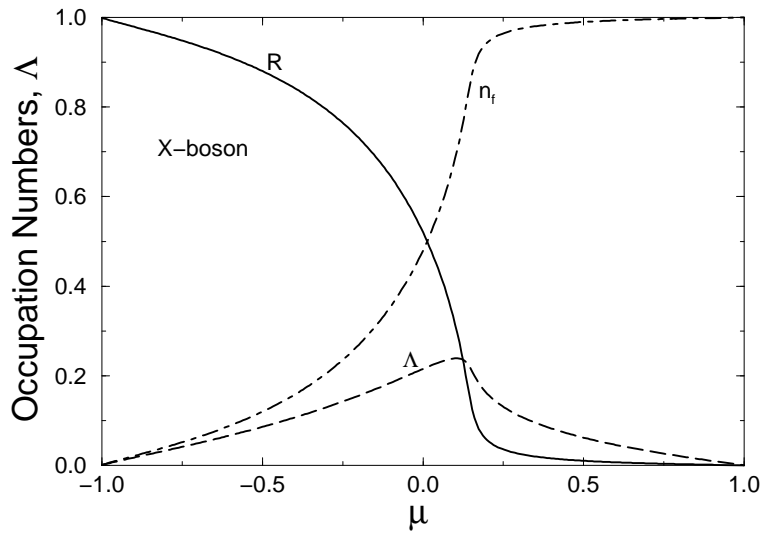

FIG. 5. X-boson: $\Lambda, R$, and $n_{f}$ vs. $\mu$ for the same model parameters used in Figure 4 . 
The values of the $\mathrm{f}$ and $\mathrm{c}$ electron density of states $\rho_{f}(\mu)$, and $\rho_{c}(\mu)$ at $\omega=\mu$ are shown as a function of $\mu$ for the slave boson method in Figure 6 and for the Xboson approach in Figure 6. The slave boson plot breaks down at $\mu=\mu_{0}$ ( where $n_{f} \rightarrow 1$ ) while for the X-boson the density of states $\rho_{f}(\mu)$ is maximum in the Kondo region and goes to zero when $\mu>>E_{f}$. In both cases one observes the transfer of conduction electrons to the $f$ band giving rise to the Kondo resonance.

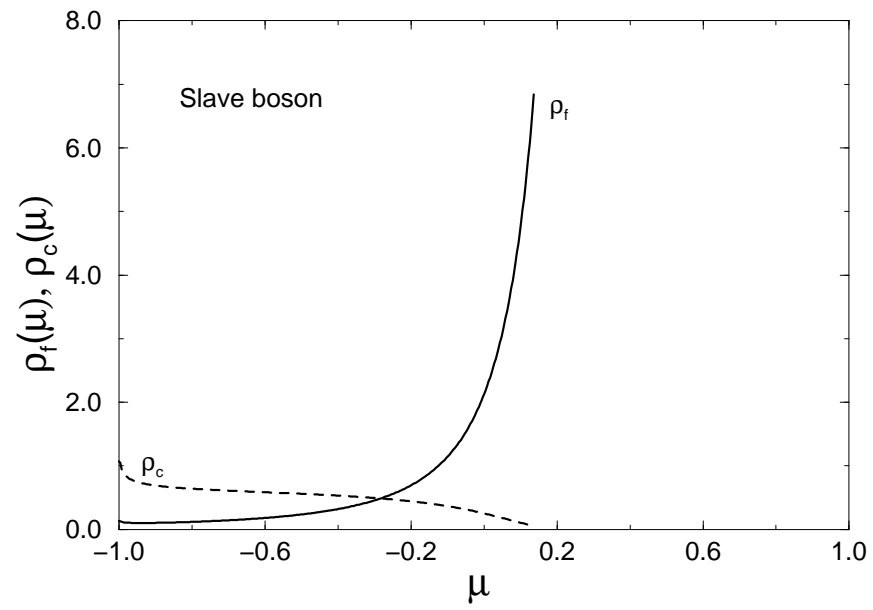

FIG. 6. Slave boson: $\rho_{f}(\mu), \rho_{c}(\mu)$ vs. $\mu$ for the same model parameters used in Figure 4 .

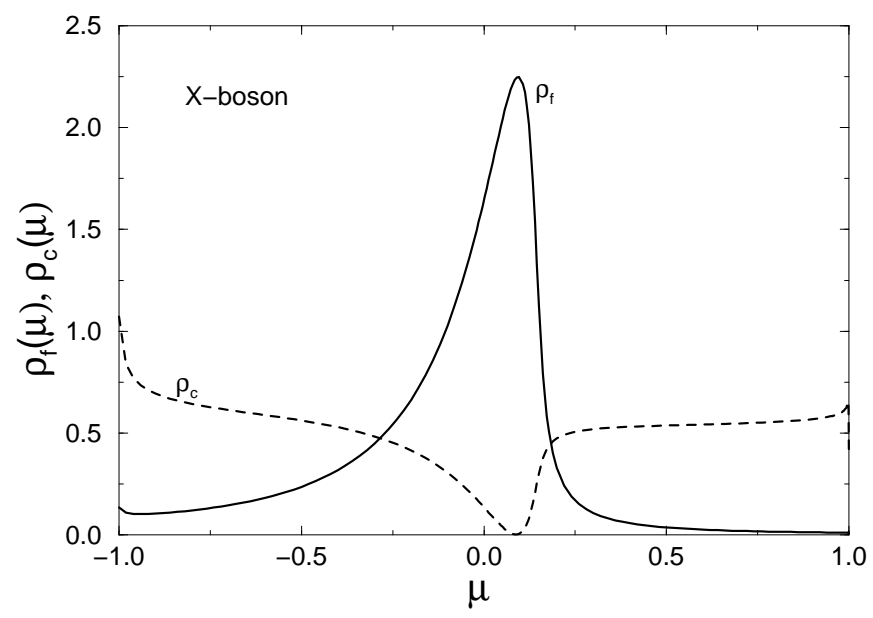

FIG. 7. X-boson: $\rho_{f}(\mu), \rho_{c}(\mu)$ vs. $\mu$ for the same model parameters used in Figure 4 .

In Figure 8 we present the density of states $\rho_{f}(\omega)$ and $\rho_{c}(\omega)$ vs. energy $\omega$ in a typical Kondo situation, both for the slave boson and X-boson treatments. The Kondo resonance appears in the two cases with a similar shape, but it is less pronounced in the X-boson treatment.

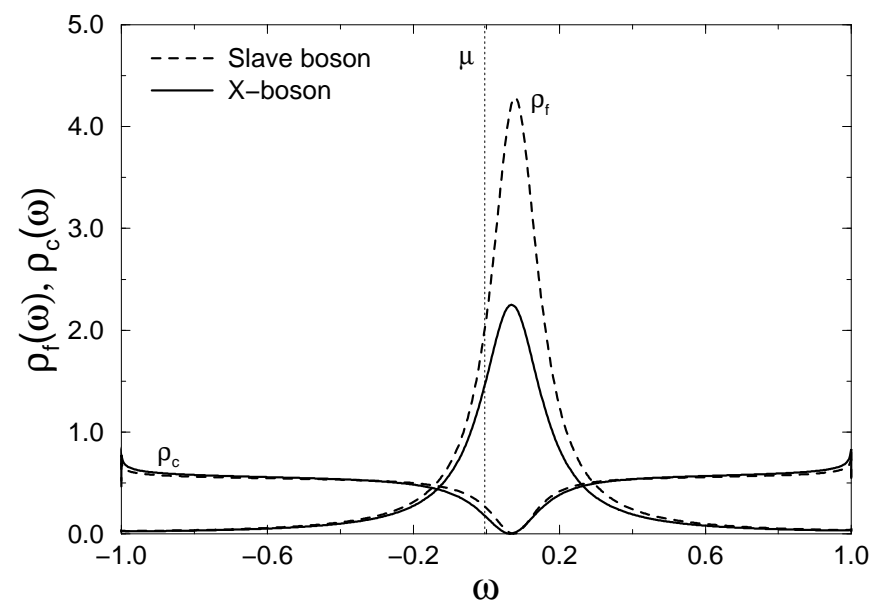

FIG. 8. Density of states of $\rho_{f}(\omega), \rho_{c}(\omega)$ vs. $\omega$ in a typical Kondo situation, in the two approaches, for the same model parameters used in Figure 1 and $\mu=0.0$.

\section{THE LATTICE PROBLEM}

For the lattice we follow the same technique employed with the impurity: the parameter $\Lambda$ is obtained by minimizing the thermodynamic potential $\Omega=-k_{B} T \ln (\mathcal{Q})$ with respect to $R$ and $D_{\sigma}=R+n_{f \sigma}$ is calculated selfconsistently. The $\Omega$ is again obtained from Eq. (32) with adequate values of $\Omega_{0}$ and $<H_{1}(\xi)>_{\xi}$ :

$$
\begin{aligned}
& \Omega_{o}=-\frac{2}{\beta} \sum_{\mathbf{k}} \ln \left[1+\exp \left(-\beta \varepsilon_{\mathbf{k}}\right)\right]+ \\
& -\frac{N_{s}}{\beta} \ln \left[1+2 \exp \left(-\beta \widetilde{\varepsilon}_{f}\right)\right]+N_{s} \Lambda(R-1), \\
& \left\langle H_{1}\right\rangle_{\xi}=\frac{1}{\pi} \int_{-\infty}^{\infty} d \omega n_{F}(\omega) \\
& \times \sum_{\mathbf{k}, \sigma} \operatorname{Im}\left\{\frac{\xi\left|V_{\sigma}(\mathbf{k})\right|^{2} D_{\sigma}}{\left(\omega^{+}-\varepsilon_{f \sigma}\right)\left(\omega^{+}-\varepsilon_{\mathbf{k} \sigma}\right)-\xi^{2}\left|V_{\sigma}(\mathbf{k})\right|^{2} D_{\sigma}}\right\},
\end{aligned}
$$

where $\omega^{+}=\omega+i 0$. As in the impurity case, this expression coincides with that obtained for an uncorrelated Hamiltonian, which in this case is Eq. (38) with a scaled hybridization $\bar{V}_{\sigma}(\mathbf{k})=\sqrt{D_{0 \sigma}} V_{\sigma}(\mathbf{k})$. The corresponding eigenvalues can be calculated analytically in this case, because for each spin component $\sigma$ the Hamiltonian is reduced into $N_{s}$ matrices $2 \times 2$. They are the poles of the lattice GFs, replacing the $N_{s}+1$ eigenvalues $\omega_{i, \sigma}$ of the impurity, and we denote them with

$$
\begin{aligned}
& \omega_{\mathbf{k}, \sigma}( \pm)=\frac{1}{2}\left(\varepsilon_{\mathbf{k}, \sigma}+\widetilde{\varepsilon}_{f}\right) \\
& \pm \frac{1}{2} \sqrt{\left(\varepsilon_{\mathbf{k}, \sigma}-\widetilde{\varepsilon}_{f}\right)^{2}+4\left|V_{\sigma}(\mathbf{k})\right|^{2} D_{\sigma}}
\end{aligned}
$$


Following the same arguments employed for the impurity we obtain

$$
\begin{aligned}
\Omega= & \bar{\Omega}_{0}+\frac{-1}{\beta} \sum_{\mathbf{k}, \sigma, \ell= \pm} \ln \left[1+\exp \left(-\beta \omega_{\mathbf{k}, \sigma}(\ell)\right]\right. \\
& +N_{s} \Lambda(R-1)
\end{aligned}
$$

where

$$
\bar{\Omega}_{0}=-\frac{N_{s}}{\beta} \ln \left[\frac{1+2 \exp \left(-\beta \widetilde{\varepsilon}_{f}\right)}{\left(1+\exp \left(-\beta \widetilde{\varepsilon}_{f}\right)\right)^{2}}\right] .
$$

The same result was obtained by direct analytical integration, thus confirming the arguments employed in the derivation of Eqs. (41,51). As in the impurity case, all the correlation effects on the thermodynamic potential appear in the $\Omega_{o}$, and one expects again a Fermi liquid behavior of the quasiparticles in the X-boson approximation (cf. the Appendix).

As in the impurity case we obtain an equation for $\Lambda$ by minimizing $\Omega$ with respect of $R$ :

$$
\begin{aligned}
\Lambda= & \frac{1}{N_{s}} \sum_{\mathbf{k}, \sigma}\left|V_{\sigma}(\mathbf{k})\right|^{2} \\
& \times \frac{n_{F}\left(\omega_{\mathbf{k}, \sigma}(+)\right)-n_{F}\left(\omega_{\mathbf{k}, \sigma}(-)\right)}{\sqrt{\left(\varepsilon_{\mathbf{k}, \sigma}-\widetilde{\varepsilon}_{f, \sigma}\right)^{2}+4\left|V_{\sigma}(\mathbf{k})\right|^{2} D_{\sigma}}},
\end{aligned}
$$

where $n_{F}(x)$ is the Fermi-Dirac distribution. Employing $\left|V_{\sigma}(\mathbf{k})\right|=V$ as well as a conduction band of constant density of states and width $W=2 D$, we find

$$
\Lambda=\frac{V^{2}}{D} \int_{-D}^{D} d \varepsilon_{\mathbf{k}} \frac{n_{F}\left(\omega_{\mathbf{k}}(+)\right)-n_{F}\left(\omega_{\mathbf{k}}(-)\right)}{\sqrt{\left(\varepsilon_{\mathbf{k}}-\widetilde{\varepsilon}_{f}\right)^{2}+4 V^{2} D_{\sigma}}} .
$$

The quasiparticle properties of a heavy fermion system can be described by an effective Hamiltonian, characterized by two hybridized bands coupled by an effective hybridization matrix element $\bar{V} .9$ In the slave boson method

$$
\bar{V}_{S-b}^{2}=\left(1-n_{f}\right) V^{2},
$$

while in the present approach we have

$$
\bar{V}_{X-b}^{2}=D_{\sigma} V^{2}=\left(1-n_{f} / 2\right) V^{2} .
$$

The hybridization reduction factor is related to the effective probability that a $c$ electron jumps into an $f$ state, and when $U \rightarrow \infty$ this transition can only take place if the $f$ level of the final site is empty. Rice and Ueda 20 argued that the effective hybridization should be

$$
\bar{V}_{R U}^{2}=\frac{1-n_{f}}{1-n_{f} / 2} V^{2},
$$

and variational calculations were performed by themen and by Fazekas.21.22 These variational calculations present the same spurious phase transitions shown by the slave-boson method, because in all these treatments the reduction factor goes to zero at a critical temperature. On the other hand, the reduction factor does not vanish in the whole range of temperatures for the X-boson approach, and the $f$ and $c$ electrons do not decouple. All these approaches produce similar results at low temperatures.

\section{LATTICE RESULTS}

We have employed for the lattice the same system parameters used to discuss the impurity, and a similar type of behavior is observed. The evolution of the X-boson parameters $R$, and $n_{f}$ are shown in Figure 9 as a function of $T$, and the $f$ and $c$ electrons do not decouple in this treatment because the hole occupation $R>0$ in the whole range of temperatures, while the corresponding parameter $r^{2}$ of the usual slave boson treatment vanishes at a critical temperature (Kondo temperature $T_{K}$ ).

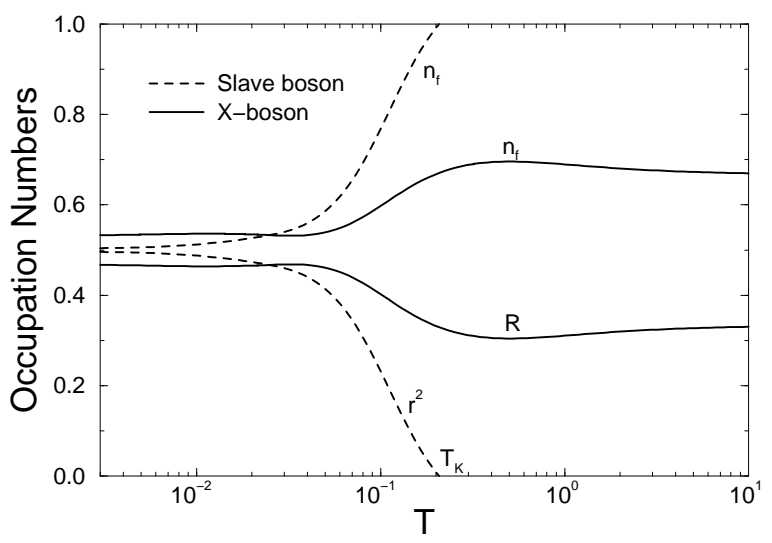

FIG. 9. Kondo case: Occupation numbers $n_{f}$, and parameters $r^{2}$ and $R$ vs. $T$ for both the slave boson and the X-boson methods, for the same model parameters used in Figure 2.

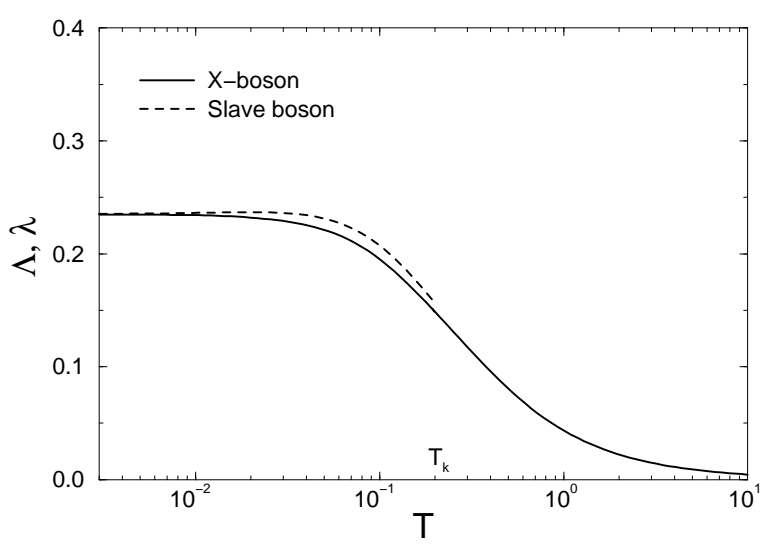

FIG. 10. Kondo case: $\Lambda$, and $\lambda$ vs. $T$ for the same model parameters used in Figure 9 . 
The two approaches yield similar results at low temperatures. The dependence with temperature of $\lambda$ (slave boson) and $\Lambda$ (X-boson) is presented in Figure 10, and the slave boson $\lambda$ breaks down at the Kondo temperature $T_{K}$ whereas the X-boson $\Lambda$ goes continuously to zero, recovering the CHA behavior at high temperatures.

The evolution of $n_{f}, \lambda$ and $r^{2}$, with the chemical potential $\mu$, is presented in Figure 11 for the slave boson treatment. In this case we do not recover the three characteristics regimes of the PAM: Kondo, intermediate valence and magnetic, because the formalism breaks down when $n_{f} \rightarrow 1$. The corresponding quantities in the $\mathrm{X}$ boson approach: $n_{f}, \Lambda$ and $R$, are also given as a function of $\mu$ in Figure 12, and the three characteristic regimes of the PAM are clearly shown. There is a plateau in this figure when the chemical potential crosses the hybridization gap, and the parameter $\Lambda$ is maximum in the Kondo region, and goes smoothly to zero together with $R$ when $\mu$ increases, while $n_{f} \rightarrow 1$. The CHA is recovered in this region by the $\mathrm{X}$-boson treatment.

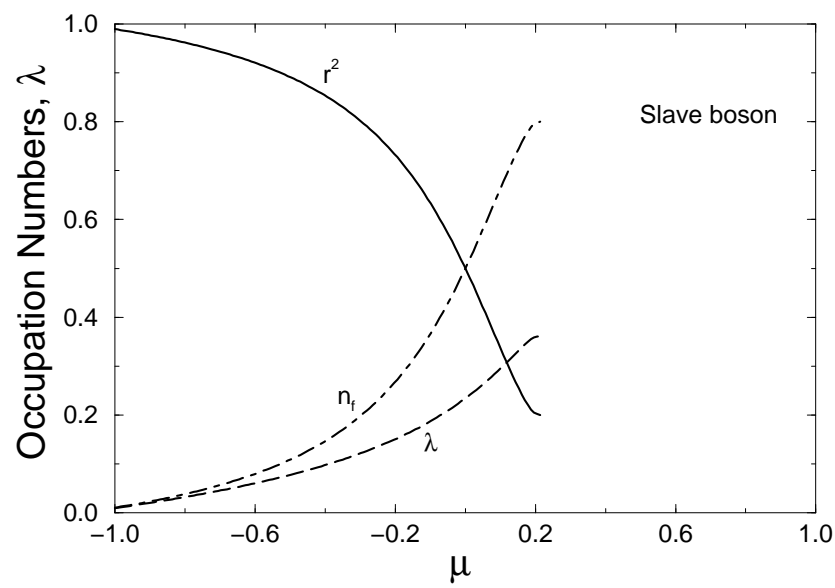

FIG. 11. Slave boson: Occupation numbers, $\lambda, r^{2}$, and $n_{f}$ vs. $\mu$ for the same model parameters used in Figure 4 .

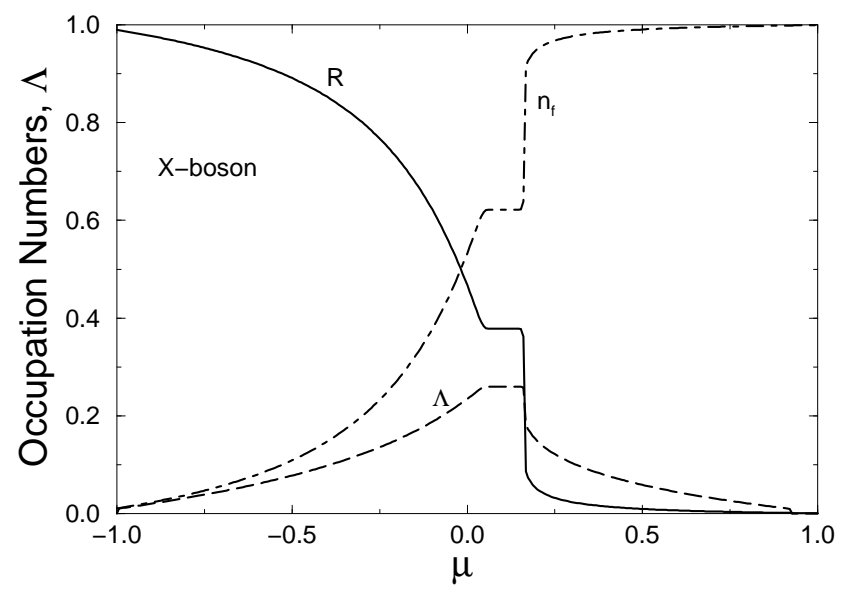

FIG. 12. X-boson: Occupation numbers, $\Lambda, R$, and $n_{f}$ vs. $\mu$ for the same model parameters used in Figure 1 .
The value of the density of states $\rho_{f}(\mu), \rho_{c}(\mu)$ as function of $\mu$ is presented in Figure 13 for the slave boson treatment: the approach breaks down in the Kondo region when $n_{f} \rightarrow 1$ and the same quantities are plotted in Figure 14 for the X-boson approach.

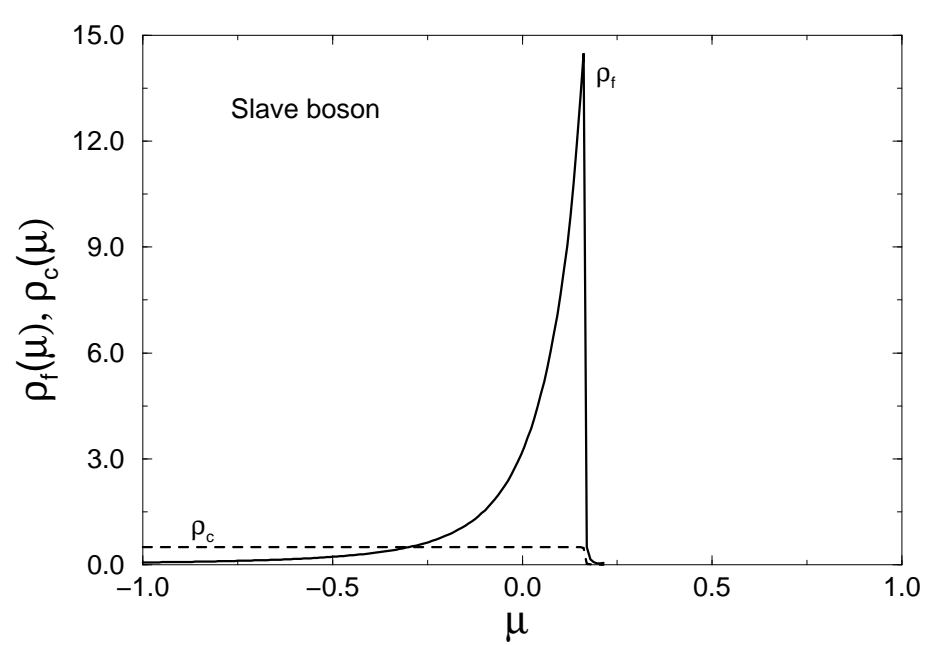

FIG. 13. Slave boson: $\rho_{f}(\mu), \rho_{c}(\mu)$ vs. $\mu$ for the same model parameters used in Figure 4 .

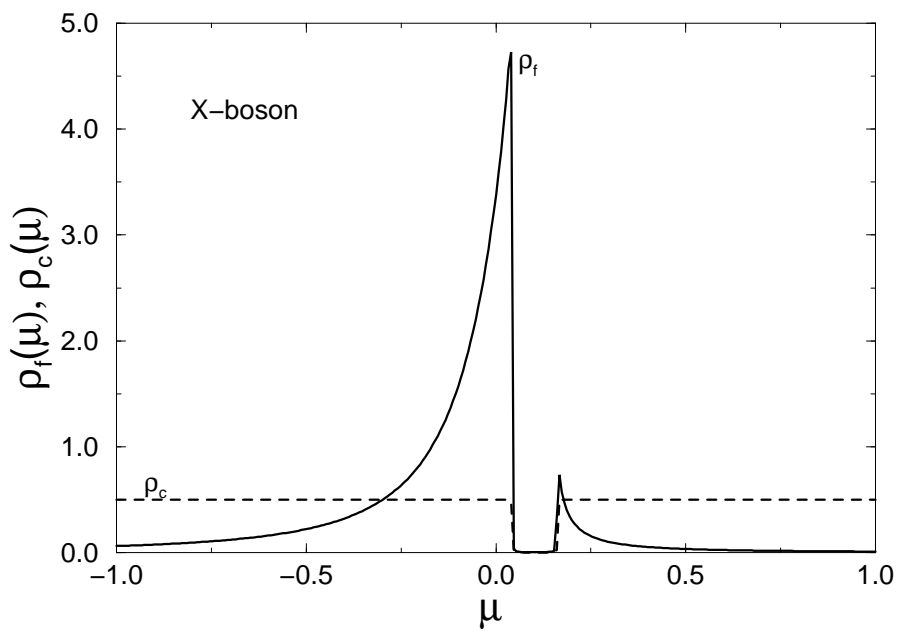

FIG. 14. X-boson: $\rho_{f}(\mu), \rho_{c}(\mu)$ vs. $\mu$ for the same model parameters used in Figure 1 .

The density of states $\rho_{f}(\omega), \rho_{c}(\omega)$ vs $\omega$ are shown in Figure 15 for both the slave boson and X-boson approaches in a typical Kondo situation. The density of $f$ states at $\mu$ is practically the same in the two cases. 


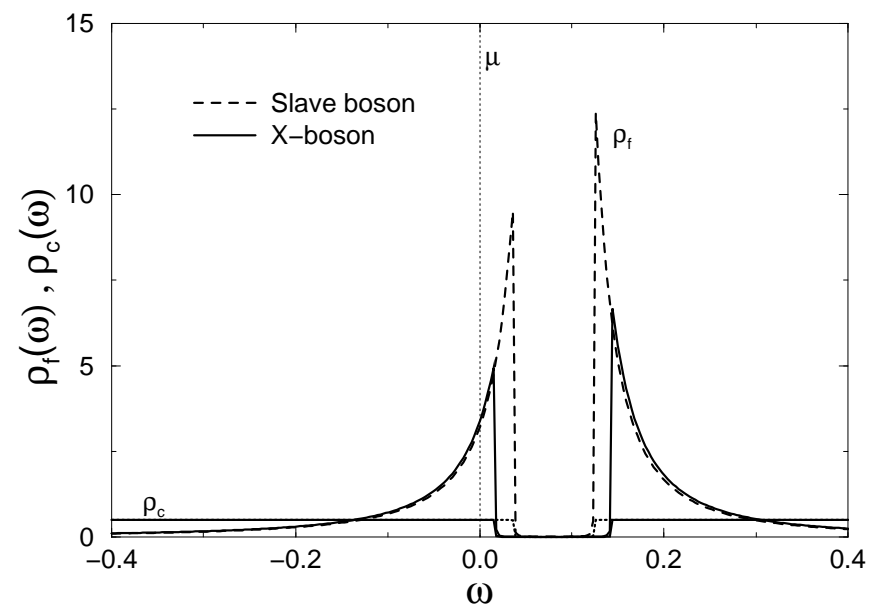

FIG. 15. Density of states in a typical Kondo situation in the two approaches: $\rho(\omega)$ vs $\omega$ for the same model parameters used in Figure and $T=0.001$.

\section{SPECIFIC HEAT}

In this section we apply the theory developed in this work to calculate the specific heat of the Kondo insulators, but for simplicity, we shall not try to fit the experimental results of some particular compound. This class of materials has been extensively studied in the last decade since its characterization by G. Aeppli and Z. Fisk 23 as Kondo insulators. Some of the compounds that we can include in this family are: $\mathrm{FeSi} ; \mathrm{Ce}_{3} \mathrm{Bi}_{4} \mathrm{Pt}_{3} ; \mathrm{SmB}_{6}$, and $\mathrm{YbB}_{12}$. The slave-boson was applied to Kondo insulators initially by P. Riseborough 24 and by Carlos Sanchez-Castro et al,25 and we have recently studief the Kondo insulator FeSi using the atomic model.26.27 We have been able to adjust simultaneously the static conductivity, the resistivity and the dynamical conductivity to the experimental results, and we obtained a fair agreement. In this section, we present the X-boson formalism as an alternative that gives results close to those obtained by the slave boson method in its region of validity, while it makes possible to extend the results to the whole range of temperatures, without presenting the spurious phase transitions appearing in the usual slave-boson method.

To calculate the specific heat employing $\Omega$ we first show by standard thermodynamic techniques that

$$
S=-\left(\frac{\partial F}{\partial T}\right)_{N, V}=-\left(\frac{\partial \Omega}{\partial T}\right)_{\mu, V}
$$

and then that

$$
\begin{aligned}
C_{v}= & T\left(\frac{\partial S}{\partial T}\right)_{N, V}=-T\left(\frac{\partial^{2} \Omega}{\partial T^{2}}\right)_{\mu, V} \\
& +T\left(\frac{\partial \mu}{\partial T}\right)_{N, V}\left(\frac{\partial N}{\partial T}\right)_{\mu, V} .
\end{aligned}
$$

Assuming a conduction band with constant density of states and width $W=2 D$ and the absence of magnetic field, we find from Eqs. (51,52) that

$$
\begin{aligned}
& -T\left(\frac{\partial^{2} \Omega}{\partial T^{2}}\right)_{\mu, V}=-T\left(\frac{\partial^{2} \bar{\Omega}_{0}}{\partial T^{2}}\right)_{\mu, V} \\
& +\frac{k_{B} \beta^{2}}{D} \sum_{\ell= \pm}^{2} \int_{-D}^{D} d x \omega_{\ell}^{2}(x) n_{F}\left(\omega_{\ell}(x)\right)\left[1-n_{F}\left(\omega_{\ell}(x)\right)\right] \\
& -T N_{s}\left(\frac{\partial^{2}(\Lambda(R-1))}{\partial T^{2}}\right)_{\mu, V}
\end{aligned}
$$

where

$$
\omega_{ \pm}(x)=\frac{1}{2}\left(x+\widetilde{\varepsilon}_{f}\right) \pm \frac{1}{2} \sqrt{\left(x-\widetilde{\varepsilon}_{f}\right)^{2}+4|V|^{2} D_{\sigma}}
$$

and

$$
\begin{gathered}
-T\left(\frac{\partial^{2} \bar{\Omega}_{0}}{\partial T^{2}}\right)_{\mu, V}=-2 N_{s} k_{B} \beta^{2} \widetilde{\varepsilon}_{f}^{2} \exp \left(\beta \widetilde{\varepsilon}_{f}\right) \\
\times \frac{\left[3+2 \exp \left(\beta \widetilde{\varepsilon}_{f}\right)\right]}{\left[\exp \left(\beta \widetilde{\varepsilon}_{f}\right)+2\right]^{2}\left[\exp \left(\beta \widetilde{\varepsilon}_{f}\right)+1\right]^{2}} .
\end{gathered}
$$

We compare the specific heat obtained by the slave boson vs. X-boson methods employing in the two cases the same parameters, corresponding to a typical Kondo insulator situation with the chemical potential inside the gap. In Figure 16 we present the corresponding density of states $\rho_{f}(\omega)$ vs. $\omega$, for the following parameters: $E_{f}=$ $0.3 ; V=0.35 ; W=2.0 ; T=0.001$ and $\mu=0.5$.

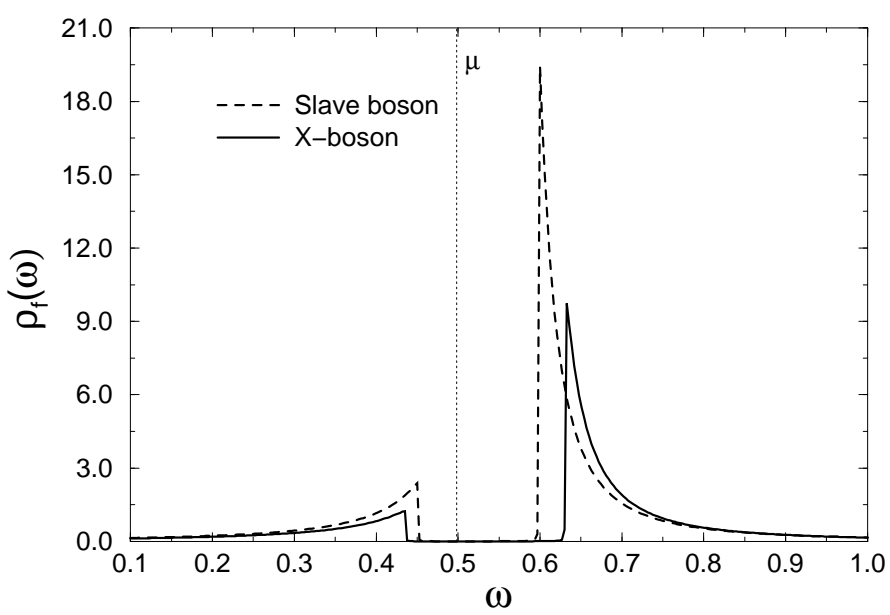

FIG. 16. Density of states in a typical Kondo insulator situation in the two approaches: $\rho_{f}(\omega)$ vs. $\omega$ with the following parameters: $E_{f}=0.3 ; W=2.0 ; V=0.35 ; \mu=0.5$; $T=0.001$.

In Figure 17 we present $C_{v}$ vs. $T$, employing the same parameters of Figure 16. We have calculated $T(\partial \mu / \partial T)_{N, V}(\partial N / \partial T)_{\mu, V}$ numerically, and its contribution to $C_{V}$ is negligible for these parameters. The calculation above the Kondo temperature $T_{K}$ in the slave 
boson case was performed for the phase of uncoupled $f$ and $c$ electrons, and the specific heat presents a discontinuity at the transition $T_{K}$, but the curve obtained by the $\mathrm{X}$-boson method is continuous.

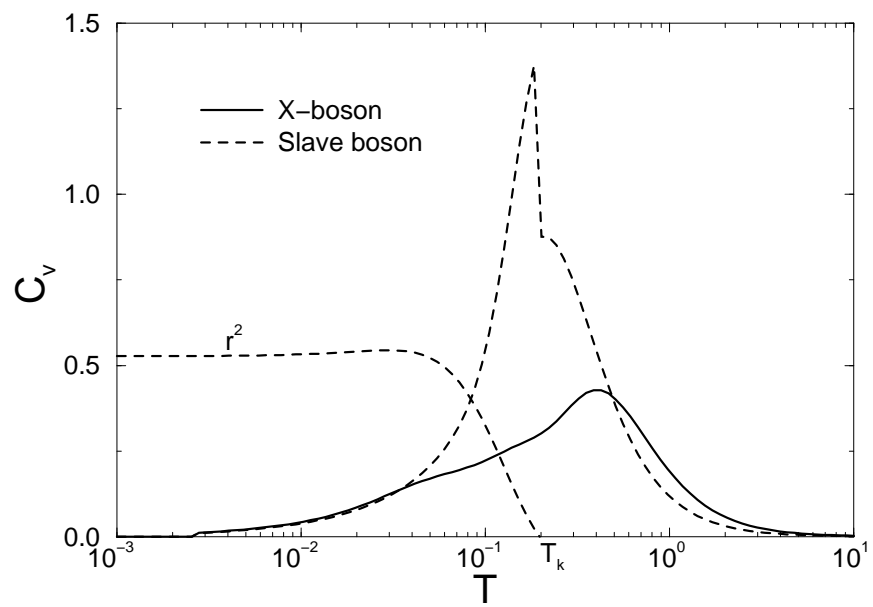

FIG. 17. Kondo insulator: Specific heat of a typical Kondo insulator for the same model parameters used in Figure 16 but as function of temperature.

\section{CONCLUSIONS}

The slave-boson formalism has heen extensively used in the mean-field approximation 1214 to study strongly correlated electron systems, because it captures the essential physics of the Kondo effect at low temperatures and its implementation in the Kondo limit is very simple. One drawback of this method is that above a temperature $T_{K}$ (cf. Section $\sqrt{\mathrm{IV}}$ ) or when $\mu>>E_{f}$, it develops a spurious second order phase transition into a phase with decoupled conduction and localized electrons. In the present work we present an approach that was inspired in some of the features of the slave-boson method in the mean-field approximation and gives similar result at low temperatures, but it does not have the spurious phase transitions of that method at higher temperatures or chemical potentials, where it behaves like the CHA solution. We have named " $X$-boson" this new technique because it keeps the $X$ operators $\theta$ even at the final stages of the calculation, while two hybridized but uncorrelated bands appear in the slave-boson method at that stage. What we employ instead is an approximation to the exact cumulant expansion of the PAM in the limit of $U \rightarrow \infty$, which is called CHA (chain approximation). 1. The CHA corresponds to the most general set of diagrams with only second order cumulants and has interesting properties like being $\Phi$-derivable. We have developed the X-boson method both for a single local impurity and for a lattice. The correlation appears in this approximation only through the presence of a constant $D_{0 \sigma}$ at critical places of the corresponding GF (cf. Eqs. (19-24, 25-30)), but it has profound effect on the system description, forcing the local electron occupation to be $n_{f}<1$, and eliminating the spurious phase transitions of the slave-boson method.

From the computational point of view the X-boson method is equivalent to the slave-boson technique, and the possibility of using it for all temperatures or chemical potentials $\mu$ makes it useful as the starting point to study several interesting problems: competition between Kondo effect and RKKY interaction in heavy fermion systems, 230 impurity bands in Kondo insulatgrs, 31 non Fermi-liquid behavior in disprdered systems, 22 and Kondo effect in quantum dots.33. 34 To give an example we have considered a Kondo insulator and calculated its specific heat: the slave boson method shows a discontinuity at $T_{K}$, while it is continuous in the X-boson method. Although it is $C_{p}$ that is usually measured, the difference $C_{p}-C_{v}$ is usually small in liquids and solids, and shows a dependence with $T$ similar to that obtained with the Xboson method (see for example, the $C_{p}$ of $\mathrm{FeSi}$ measured by Jacarino and coworkers 35 ).

In conclusion, we have presented an approach that gives essentially the same results as Coleman's slaveboson treatment at low temperatures, but without the spurious phases transitions at intermediate temperatures $T$ or large values of $\mu$. The X-boson results approach those of the cumulant expansion in the chain approximation for large values of $T$ or $\mu$.

Acknowledgments. We acknowledge Profs. E. Miranda (UNICAMP) and E. V. Anda (PUC-RJ) for helpful discussions and the financial support from São Paulo State Research Foundation (FAPESP) and National Research Council (CNPq).

\section{APPENDIX I: FERMI LIQUID BEHAVIOR OF THE X-BOSON METHOD}

We show here that the PAM has Fermi liquid properties in the $\mathrm{X}$ boson description, and to discuss the problem we shall first consider the $f$ and $c$ electrons selfenergies $\Sigma_{f}(\mathbf{k}, z)$ and $\Sigma_{c}(\mathbf{k}, z)$, that are related to the exact $\mathrm{GF}$ by the equations

$$
\begin{aligned}
G_{\mathbf{k} \sigma}^{f f}(z) & =\frac{-1}{\left(z-\widetilde{\varepsilon}_{f, \sigma}-\Sigma_{f}(\mathbf{k}, z)\right)}, \\
G_{\mathbf{k} \sigma}^{c c}(z) & =\frac{-1}{\left(z-\varepsilon_{\mathbf{k}, \sigma}-\Sigma_{c}(\mathbf{k}, z)\right)},
\end{aligned}
$$

where we employ the $z$ with the $\mu$ already subtracted (cf. Eqs.(10,11)). Employing an effective cumulant26,27 $M_{2, \mathbf{k} \sigma}^{e f f}(z)$ it is possible to write the exact GF

$$
G_{\mathbf{k} \sigma}^{f f}(z)=\frac{M_{2, \mathbf{k} \sigma}^{e f f}(z)}{1-|V(\vec{k})|^{2} G_{c, \mathbf{k} \sigma}^{o}(z) M_{2, \mathbf{k} \sigma}^{e f f}(z)}
$$


and

$$
\begin{aligned}
G_{\mathbf{k} \sigma}^{c c}(z) & =\frac{G_{c, \mathbf{k} \sigma}^{o}(z)}{1-|V(\mathbf{k})|^{2} G_{c, \mathbf{k} \sigma}^{o}(z) M_{2, \mathbf{k} \sigma}^{e f f}(z)} \\
& =\frac{-1}{z-\varepsilon_{\mathbf{k} \sigma}+|V(\mathbf{k})|^{2} M_{2, \mathbf{k} \sigma}^{e f f}(z)},
\end{aligned}
$$

where

$$
G_{c, \mathbf{k} \sigma}^{o}(z)=\frac{-1}{\left(z-\varepsilon_{\mathbf{k} \sigma}\right)}
$$

is the unperturbed GF of the $c$ electrons. The exact self energies are then

$$
\Sigma_{f}(\mathbf{k}, z)=z-\widetilde{\varepsilon}_{f, \sigma}+\left[M_{2, \mathbf{k} \sigma}^{e f f}(z)\right]^{-1}-|V(\mathbf{k})|^{2} G_{c, \mathbf{k} \sigma}^{o}(z)
$$

and

$$
\Sigma_{c}(\mathbf{k}, z)=-|V(\mathbf{k})|^{2} M_{2, \mathbf{k} \sigma}^{e f f}(z),
$$

and to recover the CHA self energies it is sufficient to replace $\mathbf{l}$ the effective cumulant $M_{2, \mathbf{k} \sigma}^{\text {eff }}(z)$ by the contribution of its simplest diagram, namely the unperturbed GF of the $f$ electrons:

$$
M_{2, \mathbf{k} \sigma}^{e f f}(z) \rightarrow G_{f, 0 \sigma}^{o}(z)=-D_{0 \sigma} /\left(z-\varepsilon_{\sigma}\right) .
$$

The essential property of a Fermi liquid is that at low temperatures it has a behavior close to that of a system of free Fermions. It is described by a system of quasiparticles that replace the elementary excitations of the free system, but they have a finite life-time caused by the interactions that are absent in the free system. Instead of the elementary particle energies we have to analyze the poles of the relevant GF, given in our problem by the solutions $z_{\mathbf{k} \sigma}$ of the equation

$$
z-\varepsilon_{\mathbf{k} \sigma}+|V(\mathbf{k})|^{2} M_{2, \mathbf{k} \sigma}^{e f f}(z)=0 .
$$

The $\operatorname{Re}\left[z_{\mathbf{k} \sigma}\right]$ corresponds to the energies of the elementary excitations, while their imaginary parts give their decay properties, and the Fermi surface is given by the set of $\mathbf{k}_{F}$ such that $\operatorname{Re}\left[z_{\mathbf{k} \sigma}\right]=0$, because we already have subtracted the $\mu$ from the $z$. The $M_{2, \mathbf{k} \sigma}^{e f f}(z)$ in the numerator of Eq. (3) does not introduce new poles, because $G_{\mathbf{k} \sigma}^{f f}(z)$ is finite at those poles.

¿From Eq. (9) we obtain

$$
M_{2, \mathbf{k} \sigma}^{e f f}\left(z_{\mathbf{k} \sigma}\right)=-\frac{z_{\mathbf{k} \sigma}-\varepsilon_{\mathbf{k} \sigma}}{|V(\mathbf{k})|^{2}},
$$

and replacing in Eq. (6) we find

$$
\Sigma_{f}\left(\mathbf{k}, z_{\mathbf{k} \sigma}\right)=z_{\mathbf{k} \sigma}-\widetilde{\varepsilon}_{f, \sigma},
$$

while from Eqs. (89) we obtain

$$
\Sigma_{c}\left(\mathbf{k}, z_{\mathbf{k} \sigma}\right)=z_{\mathbf{k} \sigma}-\varepsilon_{\mathbf{k} \sigma} .
$$

It then follows that

$$
\operatorname{Im}\left[\Sigma_{c}\left(\mathbf{k}, z_{\mathbf{k} \sigma}\right)\right]=\operatorname{Im}\left[\Sigma_{f}\left(\mathbf{k}, z_{\mathbf{k} \sigma}\right)\right]=\operatorname{Im}\left[z_{\mathbf{k} \sigma}\right]
$$

An essential property of the Fermi liquid is that the quasi-particles on the Fermi surface have an infinite lifetime when $T \rightarrow 0$, and this property follows from Eq. (12) for both the $f$ and $c$ electrons when the poles on the Fermi surface are real, because the corresponding selfenergies are then also real.

¿From Eq. (50) we conclude that the $G F$ poles of the PAM in the CHA are all real, and the same property holds for the X-boson solution. These two approximations therefore describe the PAM as a Fermi liquid, because they satisfy the condition above. Because the infinite lifetime is valid for all $\mathbf{k}$ and for all $T$, the quasiparticles in these two approximations behave more like elementary excitations than like the usual quasi-particles, that have a finite lifetime outside the Fermi surface, even at $T \rightarrow 0$.

1 M. S. Figueira, M. E. Foglio and G. G. Martinez, Phys. Rev. B 50, 17933 (1994)

2 J. Hubbard, Proc. R. Soc. London, Ser. A 285, 542 (1965); A 296, 82 (1966)

3 M. E. Foglio, M. S. Figueira, J. Phys. A: Math. Gen. 30 7879 (1997)

4 M. E. Foglio, M. S. Figueira, International Journal of Modern Physics B 12, 837 (1998)

5 A. C. Hewson, J. Phys. C: Solid State Phys. 10, 4973 (1977)

6 E. V. Anda, J. Phys. C: Solid State Phys. 14, L1037 (1981)

7 G. Baym and L. P. Kadanoff, Phys. Rev. 124, 287 (1961)

8 M. S. Figueira and M. E. Foglio, J. Phys.: Condens. Matter 8, 5017 (1996)

9 P. Coleman, Phys. Rev. B 29, 3035 (1984)

10 P. Coleman, J. Magn. Magn. Mat, 47\&48, 323 (1985)

11 R. Franco, M. S. Figueira and M. E. Foglio, J. Magn. Magn. Mat, 226\&230, 194 (2001)

12 D. M. Newns, N. Read, Advances in Physics, 36, 799 (1987)

${ }^{13}$ M. E. Foglio, Phys. Rev. B 43, 3192 (1991)

14 A. C. Hewson, The Kondo Problem to Heavy Fermions Cambridge Studies in Magnetism - Cambridge University Press, (1993)

15 M. S. Figueira, M. E. Foglio, Physica A 208 (1994)

16 A. A. Abrikosov, L. P. Gorkov and I. E. Dzyaloshinski, Quantum Field Theoretical Methods in Statistical Physics (Pergamon, Oxford), (1965)

17 S. Doniach and E. H. Sondheimer, Green's Functions for Solid State Physicists (Benjamin, New York), (1974)

18 B. H. Bernhard, C. Lacroix, J. R. Iglesias, and B. Coqblin, Physica B 259-261, 227 (1999); Ben Hur Bernhard, Claudine Lacroix, Phys. Rev. B60, 12149 (1999) 
19 Peter Fulde, Joachim Keller, Gertrud Zwickngl, Solid State Physics 41, 1 (1988)

20 T. M. Rice and K. Ueda, Phys. Rev. B Condens. Matter 34, 6420 (1986)

21 P. Fazekas, J. Magn. Magn. Mat. 63\&64, 545 (1987)

22 Patrik Fazekas, Lecture Notes on Electron Correlation and Magnetism - Series in Modern Condensed Matter Physics - Vol. 5 - World Scientific Publishing Co. (1999)

23 G. Aeppli and Z. Fisk, Comments Cond. Mat. Phys. 16, 155 (1992)

24 Peter S. Riseborough, Phys. Rev. B 45, 13984 (1992)

25 Carlos Sanchez-Castro, Kevin S. Bedell and Bernard R. Cooper, Phys. Rev. B 47, 6879 (1993)

26 M. E. Foglio and M. S. Figueira, Phys. Rev. B 60, 11361 (1999)

27 M. E. Foglio and M. S. Figueira, Phys. Rev B 62, 7882 (2000)

28 J. R. Iglesias, C. Lacroix and B. Coqblin, Phys. Rev B 56, 11820 (1997)

${ }^{29}$ T. G. Rappoport, M. S. Figueira, M. A. Continentino, Physics Letters A 264, 497 (2000)

30 A. H. Castro Neto and B. A. Jones, Phys. Rev B 62, 14975 (2000)

31 P. Schlottmann, Phys. Rev B 46, 998 (1992)

32 E. Miranda, V. Dobrosavljević and G. Kotliar, J. Phys.: Condens. Matter 8, 9871 (1996)

33 Luis Craco and Kicheon Kang, 59, 12244 (1999)

34 V. Ferrari, G. Chiappe, E. V. Anda and Maria A. Davidovich, Phys. Rev. Letters 82, 5088 (1999)

35 V. Jaccarino, G. K. Wertheim, J. H. Wernick, L. R. Walker, Sigurds Arajs, Phys. Rev. B 160, 476(1967) 